Bull. Austral. Math. Soc.

Vol. 41 (1990) [343-346]

\title{
FORMAL COMPLEXITY OF INVERSE SEMIGROUP RINGS
}

\author{
Adel A. Shehadah
}

A ring $\left(R,{ }^{*}\right)$ with involution * is called formally complex if $\sum A_{i} A_{i}^{*}=0$ implies that all $A_{i}$ are 0 . Let $\left(R,{ }^{*}\right)$ be a formally complex ring and let $S$ be an inverse semigroup. Let $\left(R[S],{ }^{*}\right)$ be the semigroup ring with involution * defined by $\left(\sum a_{i} s_{i}\right)^{*}=\sum a_{i}^{*} s_{i}^{-1}$. We show that $\left(R[S],{ }^{*}\right)$ is a formally complex ring. Let $\left(S,{ }^{*}\right)$ be a semigroup with proper involution ${ }^{*}\left(a a^{*}=a b^{*}=b b^{*} \Rightarrow a=b\right)$ and let $\left(R, *^{\prime}\right)$ be a formally complex ring. We give a sufficient condition for $\left(R[S],{ }^{*}\right)$ to be a formally complex ring and this condition is weaker than * being the inverse involution on $S$. We illustrate this by an example.

Definitions: Let $\left(S,{ }^{*}\right)$ be a semigroup with involution *; that is * satisfies $(a b)^{*}=b^{*} a^{*}$ and $\left(a^{*}\right)^{*}=a$. We say that ${ }^{*}$ is proper and $\left(S,{ }^{*}\right)$ is a proper ${ }^{*}$-semigroup if $(\forall a, b \in S)\left(a a^{*}=a b^{*}=b b^{*} \Rightarrow a=b\right)$. In this case we have *-cancellation in the sense that if $a a^{*} b=a a^{*} c$ then $a^{*} b=a^{*} c$ because then $b^{*} a \cdot a^{*} b=b^{*} a \cdot a^{*} c=c^{*} a . a^{*} c$. Let $\left(R,{ }^{*}\right)$ be a ring with involution ${ }^{*}$; that is ${ }^{*}$ satisfies $(a+b)^{*}=a^{*}+b^{*},(a b)^{*}=b^{*} a^{*}$ and $\left(a^{*}\right)^{*}=a$. Then ${ }^{*}$ is formally complex and $\left(R,,^{*}\right)$ is a formally complex ring if for every finite subset $\left\{a_{1}, \ldots, a_{n}\right\}$ of distinct elements of $R, \sum a_{i} a_{i}^{*}=0$ implies that all $a_{i}$ are 0 . If for every $a$ in $R, a a^{*}=0$ implies that $a=0$, we say that * is proper and $\left(R,{ }^{*}\right)$ is a proper ${ }^{*}$-ring. Let $\left(R,{ }^{*}\right)$ be a ring with involution and let $\left(S, *^{\prime}\right)$ be a *-semigroup with involution $*^{\prime}$. We define the natural involution * on the semigroup ring $R[S]$ as follows: $\left(\sum a_{i} s_{i}\right)^{*}=\sum a_{i}^{*} s_{i}^{*^{\prime}}$. In particular, if $S$ is an inverse semigroup and $\left(R,{ }^{*}\right)$ is a *-ring, then the natural involution ${ }^{*}$ on $R[S]$ takes the form $\left(\sum a_{i} s_{i}\right)^{*}=\sum a_{i}^{*} s_{i}^{-1}$.

It is proved in [2] that if $S$ is an inverse semigroup and if $\left(R,{ }^{*}\right)$ is a formally complex ring then $\left(R[S],{ }^{*}\right)$ is a proper ${ }^{*}$-ring under the natural involution. We prove that it is formally complex. We need the following lemma proved in [2].

LEMMA . Let $S$ be an inverse semigroup and let $a, b, c \in S$ be such that $a a^{-1}=$ $b c^{-1}$. Then $a^{-1} b=a^{-1} c$.

THEOREM 1. Let $S$ be an inverse semigroup and let $\left(R,{ }^{*}\right)$ be a formally complex ring. Then $\left(R[S],{ }^{*}\right)$, under the natural involution ${ }^{*}$, is formally complex.

Received 12 May 1989

Copyright Clearance Centre, Inc. Serial-fee code: 0004-9729/90 \$A2.00+0.00. 
Proof: For $A=\sum a_{i} s_{i} \in R[S]$ we set $\operatorname{supp}(A)=\left\{s_{i} \mid a_{i} \neq 0\right\}$. Let $A_{1}, \ldots, A_{n} \in R[S]$ be such that $\sum A_{i} A_{i}^{*}=0$. Let $X=\cup \operatorname{supp}\left(A_{i}\right)$. We have to show that $X=0$. We prove the equivalent assertion that it is impossible to have $\sum A_{i} A_{i}^{*}=0$ and $0<|X| \leqslant n$, where $n \in N$. If $|X|=1$ then $A_{i}=a_{i} s$ for some fixed $s \in S$ and for $i=1, \ldots, n$. Then $0=\sum A_{i} A_{i}^{*}=\left(\sum a_{i} a_{i}^{*}\right) s s^{-1}$ which implies that $\sum a_{i} a_{i}^{*}=0$. Thus all $a_{i}$ are 0 since $R$ is formally complex, a contradiction. Assume the assertion to be true for $0<|X| \leqslant n-1$. Let $\sum A_{i} A_{i}^{*}=0$ such that $|X|=n$. We choose $s \in X$ which is maximal in the sense that $s \leqslant t$ implies that $s=t$ for all $t \in X$. Here $u \leqslant v$ means that $u v^{-1}=u u^{-1}$ which is the Vagner-Preston partial ordering on inverse semigroups. Only one of the following two cases occurs:

(1) $s s^{-1}=s_{i} s_{j}^{-1}$ for some $i \neq j$.

In this case $s_{i} \neq s \neq s_{j}$ since $s s^{-1}=s s_{i}^{-1}$ implies $s \leqslant s_{i}$ and so $s=s_{i}$.

(2) $s s^{-1}=s_{i} s_{j}^{-1}$ implies that $i=j$.

In Case (1) let $s s^{-1}=s_{i} s_{j}^{-1}$ for some $i, j, i \neq j$. This implies that $s^{-1} s_{i}=s^{-1} s_{j}$ by the lemma. But now $\left|X^{\prime}\right|=\left|\cup \operatorname{supp} s^{-1} A_{i}\right|<n$, while $\sum\left(s^{-1} A_{i}\right)\left(s^{-1} A_{i}\right)^{*}=$ $s^{-1}\left(\sum A_{i} A_{i}^{*}\right) s=0$. By induction $X^{\prime}=\emptyset$. Since $s$ occurs in some $A_{i}$ we have $s^{-1} A_{i}=0$. But then $s^{-1} s=0$ if $\operatorname{supp}\left(A_{i}\right)=\{s\}$, or there is $t \in X$ distinct from $s$ such that $s^{-1} s=s^{-1} t$. This is impossible.

In Case (2) the coefficient of $s s^{-1}$ in the expansion of $\sum A_{i} A_{i}^{*}=0$ is a sum of terms of the form $c c^{*}$ and this coefficient is zero. By the formal complexity of $\left(R,{ }^{*}\right)$ all $c$ 's involved in this coefficient are zero and so $1 \leqslant|X|<n$. This is impossible.

Let $\left(S,{ }^{*}\right)$ be a semigroup with involution * Let $A \subseteq S$. An element $t \in A$ is called maximal if

$$
(\forall u, v \in A)\left(\left(t t^{*}=t u^{*} \Rightarrow t=u\right) \&\left(\left(t t^{*}=u v^{*} \& u \neq t \neq v\right) \Rightarrow t^{*} u=t^{*} v\right)\right) .
$$

If every finite non-empty subset of $S$ has a maximal element we say that $\left(S,{ }^{*}\right)$ satisfies the maximal property and it is an $M P$-semigroup. We prove the following.

Proposition. Let $\left(S,{ }^{*}\right)$ be a proper ${ }^{*}-M P$-semigroup. Let $\left(R,{ }^{*}\right)$ be a formally complex ring. Then $\left(R[S],{ }^{*}\right)$ with the natural involution * is formally complex.

Proof: The proof is similar to that of the previous theorem. We prove the equivalent assertion that it is impossible to have $\sum A_{i} A_{i}^{*}=0$ and $1 \leqslant|X| \leqslant n$, where $n$ is any natural number. Here $X=U \operatorname{supp}\left(A_{i}\right)$. If $|X|=1$ then $A_{i}=a_{i} s$ for some fixed $s$. Then $0=\sum A_{i} A_{i}^{*}=\left(\sum a_{i} a_{i}^{*}\right) s s^{*}$ which implies that all $a_{i}$ are 0 by the formal complexity of $\left(R,{ }^{*}\right)$. This is impossible. Assume the assertion to be true for $1 \leqslant|X| \leqslant n-1$. Let $\sum_{1}^{m} A_{i} A_{i}^{*}=0$ and let $|X|=n$. We choose $s \in X$ which is maximal. We have only two cases to consider:

(1) $s s^{*}=s_{i} s_{j}^{*}$ for some $i \neq j$. 
In this case $s_{i} \neq s \neq s_{j}$ by maximality of $s$.

(2) $s s^{*}=s_{i} s_{j}^{*}$ implies that $i=j$.

In case (1) let $s s^{*}=s_{i} s_{j}^{*}$ for some $i \neq j$. This implies that $s^{*} s_{i}=s^{*} s_{j}$ by maximality of $s$. But now $\left|X^{\prime}\right|=\left|\cup \operatorname{supp}\left(s^{*} A_{i}\right)\right|<n$ while $\sum\left(s^{*} A_{i}\right)\left(s^{*} A_{i}\right)^{*}=$ $s^{*}\left(\sum A_{i} A_{i}^{*}\right) s=0$. This is impossible unless $X^{\prime}=\emptyset$. Let $X^{\prime}=\emptyset$. Then $s^{*} A_{i}=0$, $i=1, \ldots, n$. Let $B_{1}, B_{2}, B_{3}$ be elements in $\left\{A_{1}, \ldots, A_{m}\right\}$ such that $s \in B_{1}, s_{i} \in B_{2}$, $s_{j} \in B_{3}$. There exist positive integers $n_{1}, n_{2}, n_{3}$ such that if $B=n_{1} B_{1}+n_{2} B_{2}+n_{3} B_{3}$ then $s, s_{i}, s_{j} \in \operatorname{supp}(B)$. Since $s^{*} B_{i}=0$ for $i=1,2,3$ we have $s^{*} B=0$. Thus $s^{*} s=s^{*} s_{i}=s^{*} s_{j}=s_{i}^{*} s=s_{j}^{*} s, s s^{*}=s_{i} s_{j}^{*}=s_{j}^{*} s_{i}$. This implies that $s s^{*} s=s_{i} s_{j}^{*} s=s_{i} s^{*} s$. By ${ }^{*}$-cancellation $s s^{*}=s_{i} s^{*}=s s_{i}^{*}$. But then $s=s_{i}$ by maximality of $s$ and this is impossible.

REMARK. Let $\left(R,{ }^{*}\right)$ be a formally complex ring with 1 . Let $n$ be a positive integer. Let $e_{i j}$ be the $n \times n$ matrix with 1 as the $i j$-entry and 0 elsewhere, $1 \leqslant i, j \leqslant n$. Then $E=\left\{e_{i j}\right\} \cup\{0\}$ is an inverse semigroup with $e_{i j}^{-1}=e_{j i}$. Let $M_{n}(R)$ be the ring of all $n \times n$ matrices with entries in $R$. Then $M_{n}(R)=R[E]$. Let ${ }^{*}$ be the involution on $M_{n}(R)$ defined by $\left[a_{i j}\right]^{*}=\left[a_{i j}^{*}\right]^{t}$ and let ${ }^{*}$ be the natural involution on $R[E]$. Then $\left(M_{n}(R),{ }^{*}\right)=\left(R[E],{ }^{*}\right)$, a formally complex ring by the theorem. Of course this can be proved directly. The same thing can be said about finite sums of such matrix rings.

We now give an example of a formally complex ring over a semigroup which is not an inverse semigroup.

EXAMPLE. There is a finite regular ${ }^{*}$-semigroup $\left(S,{ }^{*}\right)$ which is not an inverse semigroup and such that for any formally complex ring $\left(R,{ }^{*}\right)$ the semigroup ring $\left(R[S],{ }^{*}\right)$ with the natural involution is formally complex.

Proof: Let $S$ be the set of all $2 \times 2$-matrices, with entries from $Z_{3}$, of the form $A_{n}=n e_{11}, B_{n}=n e_{22}, C_{n}=n\left(e_{11}+e_{12}+e_{21}+e_{22}\right), D_{n}=n e_{12}, D_{n}^{t}, E_{n}=$ $n e_{11}+n e_{12}, E_{n}^{t}, F_{n}=n e_{12}+n e_{22}, F_{n}^{t}, 0$. Since $n \in Z_{3}$ there are 19 elements in $S$. It is easy to check that $S$ is a semigroup. Let $*$ be the transpose mapping on $S$. We show that ${ }^{*}$ is a proper involution. Let $X, Y \in S$ be such that $X X^{t}=$ $X Y^{t}=Y Y^{t}$. Then $(X-Y)(X-Y)^{t}=0$ in the ring of $2 \times 2$-matrices in $Z_{3}$. If $X-Y=a e_{11}+b e_{12}+c e_{21}+d e_{22}$ it follows that $a^{2}+b^{2}=c^{2}+d^{2}=0$. But in $Z_{3}$ this implies that $a=b=c=d=0$. Thus $X=Y$ and $\left(S,{ }^{*}\right)$ is a proper *-semigroup.

We show that $S$ is regular. Using the fact that $n^{3}=n$ in $Z_{3}$ it is easily verified that $A_{n}^{3}=A_{n}, B_{n}^{3}=B_{n}, C_{n}^{3}=C_{n}, E_{n}^{3}=E_{n}, F_{n}^{3}=F_{n}$ and $D_{n} E_{n}^{t} D_{n}=D_{n}$. Thus $S$ is regular.

We show that $S$ is not an inverse semigroup. Let $x=e_{11}, y=e_{12}, z=e_{11}+e_{21}$. Then it is easily verified that $z y z=z, y z y=y, z x z=z, x z x=x$. Thus $z$ has two 
inverses and $S$ is not an inverse semigroup.

To show that $\left(R[S],{ }^{*}\right)$ is formally complex we notice that in $S, x x^{t}=x y^{t}$ implies that $x=y$ and $x x^{t}=u v^{t}$ implies that $u=v$. Thus if $\emptyset \neq S^{\prime} \subseteq S$ then all elements of $S^{\prime}$ are maximal and so the previous proposition applies. We conclude that $\left(R[S],{ }^{*}\right)$ is formally complex.

The semigroup $S$ can be described verbally as the set of all multiples of elementary matrices, all multiples of matrices with one zero row (column) and the other row (column) equal to $(1,1)$ and all matrices with equal entries.

Finally we prove the following:

TheOREM 2. Let $\left(S,{ }^{*}\right)$ be a proper- ${ }^{*}-M P$ semigroup and let $\left(R,{ }^{*}\right)$ be a formally complex ring. Then $R[S]$ is semisimple.

Proof: Let $A$ be an element of the radical of $R[S]$. Then $A A^{*}$ is in the radical and so there is a natural number $n$ such that $\left(A A^{*}\right)^{n}=0$. We can assume that $n=2^{k}$. Using the properness of the natural involution ${ }^{*}$ on $R[S]$ we conclude that $A A^{*}=0$ and so $A=0$.

\section{REFERENCES}

[1] M. Drazin, 'Regular Semigroups with Involution', Symposium on Regular Semigroups, (Northern Mlinois Univ. 1979), pp. 29-48.

[2] A. Shehadah, 'Proper embeddability of Inverse Semigroups', Bull. Austral. Math. Soc. 34 (1986), 383-387.

Mathematics Department

Yarmouk University

Irbid, Jordan 